\title{
Interactive Multi-Objective Design of Long-Span Trusses
}

\author{
Breanna Bailey \\ Department of Civil Engineering \\ Texas A\&M - Kingsville \\ Kingsville, TX 78363 \\ 001-361-593-2266 \\ breanna.bailey@tamuk.edu
}

\author{
Anne Raich \\ Department of Civil Engineering \\ Lafayette College \\ Easton, PA 18042 \\ 001-610-330-5590 \\ raicha@lafayette.edu
}

\begin{abstract}
The research presented develops a method for capturing user design preferences interactively and then incorporating these preferences into the multi-objective optimization of long-span trusses. A self-organizing map groups truss designs found in genetic algorithm populations based on information concerning quantifiable truss features. The user interactively selects preferred designs by examining representative truss designs from each group. A hybrid neural network trains on the user selections and is used by the multi-objective genetic algorithm to predict whether a user prefers new trusses in new truss designs. The predicted truss preferences are used in combination with structural design criteria during fitness evaluation, selection, and ranking. The addition of user preferences generates more design alternatives on the Pareto-optimal front and the designs evolved reflect the user preferences in addition to optimizing structural criteria.
\end{abstract}

\section{Categories and Subject Descriptors \\ J.2 [Computer Applications]: Physical Sciences and Engineering - engineering.}

\section{General Terms}

Design, Human Factors.

\section{Keywords}

Interactive Design, Multiobjective Optimization, Truss Topology.

\section{INTRODUCTION}

Long-span roof trusses create spaces that are aesthetically pleasing, improve visibility, and provide storage flexibility, while offering significant material and cost savings over other types of systems. Engineering effective designs for these systems, however, requires the satisfaction of design criteria beyond structural requirements. Although designers can often visually identify their preferences concerning the desired architectural and constructability features of a design, formulating these intrinsic preferences in the form of constraints that can be used for optimization is very difficult. This research effort develops a method for capturing user design preferences interactively and then incorporating these preferences into a multi-objective genetic algorithm (MOGA) program that optimizes long-span roof trusses.

Copyright is held by authors/owner(s)

GECCO'07, July 7-11, 2007, London, England, United Kingdom. ACM 978-1-59593-697-4/07/0007

\section{DESCRIPTION OF THE IRR MOGA}

The implicit redundant representation (IRR) MOGA is a useful tool for the conceptual design of long-span roof trusses. The flexibility to generate new and unusual trusses, in addition to rapidly generating and evaluating these potential designs, provides an environment in which designs can be tailored to meet the needs of a specific project [1]. The current research adds a new objective to the MOGA process: satisfaction of user preferences. An interactive approach is defined. During optimization, the MOGA asks the user to identify their preferences for the current population designs. To avoid having the user view every design, a self-organizing map groups designs based on quantifiable geometric truss features. The user interactively selects their preferred designs by examining a smaller set of representative truss designs from each group. Users select representative trusses from the classified population every five generations. After each interaction, a hybrid rough set reduct/back-propagation neural network is updated using the trusses selected by the user. The hybrid neural network predicts whether or not each new design evolved by the MOGA reflects the user's preferences. The MOGA stores the predictions with data collected about the design's weight, deflection, and constraint violations and uses this information during selection and ranking.

\section{CONCLUSIONS}

Trials were performed with and without user preferences to compare the quality of the Pareto-optimal design alternatives evolved by the MOGA. The truss designs on the Pareto-optimal fronts are defined by several distinct topology layouts, with design variations along the front corresponding to changes in member size or geometric perturbations of a topology. In general, including user preferences enabled the MOGA to evolve designs that reflected user preferences and were structurally efficient. In all trials, the addition of user preferences increased the extent and the uniformity of the Pareto-optimal front. In trials in which the user-preferred designs varied significantly from conventional truss designs, a second "front" formed in addition to the structurally-optimal front obtained in other trials, which contained design that sought the best compromise between satisfying the stated structural and preference criteria. The designs found on the second Pareto front had distinct features defined by user preferences.

\section{REFERENCES}

[1] Paik, S. Multi-Objective Optimal Design of Steel Trusses in Unstructured Design Domains. M.S. Thesis, Texas A\&M University, College Station, TX, 200 